\title{
Questions for the "Dying Brain Hypothesis"
}

\author{
William J. Serdahely, Ph.D. \\ Montana State University
}

ABSTRACT: I pose four questions for the "dying brain hypothesis" as propounded by Susan Blackmore in her book Dying to Live (1993). The first calls into question Blackmore's reductionist explanation of the "bird's-eye view' for a near-death experience (NDE) and asks why out-of-body perception from a supine position is not reported, given her theory. The second inquires as to how the materialist view explains NDErs' feelings of unconditional love, while the third ponders whether the variance among NDEs noted by Blackmore is not more consistent with the "afterlife hypothesis" than with the "dying brain hypothesis." The final question queries whether neural disinhibition, described by Blackmore, might be a possible release mechanism for an NDE. I suggest that these four questions pose a challenge to the "dying brain hypothesis."

Susan Blackmore wrote in her book Dying to Live: Near-Death Experiences (1993) that

All things considered, I can see no reason to adopt the afterlife hypothesis. . . . [F]or me the evidence and the arguments are overwhelming. The dying brain hypothesis, for all its shortcomings, does a better job of accounting for the experiences themselves. (p. 263)

Blackmore is an ardent proponent of what she called the "dying brain hypothesis," championing a reductionist explanation for near-death experiences (NDEs); while I am an advocate of what Blackmore termed the "afterlife hypothesis," a nonreductionist explanation for

William J. Serdahely, Ph.D., retired Professor of Health Science at Montana State University, is now Lecturer in Health Psychology at Silver Lake College in Manitowoc, WI, and also teaches at the University of Wisconsin-Manitowoc and the University of Wisconsin-Fox Valley. Reprint requests should be addressed to Dr. Serdahely at 837 West Fifth Street, Appleton, WI 54914. 
NDEs (Serdahely, 1992, 1995). In this article, I raise four questions that I believe pose serious challenges to the "dying brain hypothesis" as outlined in Dying to Live.

\section{The Bird's-Eye View}

First, if the out-of-body experience (OBE) is, as Blackmore claimed, due to the brain's reconstruction of memories and the possible tactile and auditory input while the NDEr is unresponsive, then why would we not expect out-of-body perceptions to occur from a supine, or for some NDErs, prone perspective? Blackmore suggested "The OBE . . . is the brain's way of dealing with a breakdown in the body image and model of reality" when near death (p. 261). OBEs then become reconstructed "memory images," which "are often built in a bird's-eye view" (Blackmore, 1993, p. 177). Blackmore cited an article by Georgia Nigro and Ulric Neisser (1983) to support the bird's-eye perspective contention. She also quoted Ronald Siegel, who gave an example of remembering walking along a seashore, and claimed that "Many people recall such scenes in a kind of bird's-eye view" (p. 177). She concluded by writing:

It seems likely, therefore, that in the event of nearly dying, or any other circumstance in which the normal model of reality has broken down, such a bird's-eye memory model may take over as 'real.' ( $p$. 177)

At last we have a simple theory of the OBE: the normal model of reality breaks down and the system tries to get back to normal by building a new model from memory and imagination. If this model is from a bird's-eye view, then an OBE takes place. In order for the dying brain hypothesis to account for OBEs on the basis of memory images and memory reconstruction, Blackmore must account for the "bird's-eye" perspective, that is, NDErs' sightings from a perspective of being above, looking down. Blackmore admitted that "If these [outof-body vision] claims are valid then the theory I am developing is wrong-or, to be more accurate, inadequate" (p. 113). So the aerial perspective for the dying brain hypothesis then rests on the works of Nigro and Neisser and of Siegel.

Nigro and Neisser (1983) reported finding two types of memories: observer memory, where one is "looking at the situation from an external vantage point and seeing oneself 'from the outside'" (p. 467), 
and field memory, where "one seems to have roughly the field of view that was available in the original position and one does not 'see oneself" " (pp. 467-468).

Nigro and Neisser warned against making too much of their studies of the point of view of memories. They wrote:

Our investigation is only a preliminary one. So far we have studied only a few situations, a few recall instructions, an unrepresentative sample of subjects, and an uncontrolled range of recencies. (1983, p. 481)

One study included 40 undergraduates, and the other "involved much younger subjects" (1983, p. 481), 30 advanced-placement high school students who participated in the study for pay and who may have exhibited "fatigue," according to Nigro and Neisser, due to the length of the questionnaire.

What these investigators actually found was that "As in earlier studies, there were more field than observer memories" (1983, p. 479). They concluded that

A deliberate attempt to remember the "objective circumstances" of an event leads to relatively more observer memories; a focus on feelings leads to more field memories. People who are given no special recall set generally focus on their own feelings in remembering an event. This tendency may help to explain the overall preponderance of $F$ [field] memories in our data. Another reason for that preponderance may be simply that recent memories tend to be in the field mode, and our open-ended instructions tended to elicit recall of recent events. It is also possible, of course, that there are simply more F [field] memories altogether. (1983, p. 481)

Nigro and Neisser commented further on the possible reason why some memories may be in the observer rather than the field mode:

Observer memories do occur. We are inclined to agree with Freud that they are often produced by a process of reconstruction in memory. We do not think that all of them originate in this way, however, because there may well be $O$ [observer] experiences as well as $O$ [observer] memories. In particular, events involving high degrees of emotional self-awareness may be experienced from an observer perspective. Our finding that such events produce a relatively high proportion of $O$ [observer] memories is consistent with that hypothesis. (1983, p. 481, original italics)

So Nigro and Neisser wrote that observer memories may be due to events that were experienced from an observer perspective. The "observer experiences" described by Nigro and Neisser resemble what 
the afterlife hypothesis would call the separation from the physical body, or an OBE.

The second source used by Blackmore to support the bird's-eye view in an OBE was Siegel's 1977 article focusing on his work giving an unspecified number of subjects various drugs of unspecified dosages and recording the subsequent hallucinations experienced by these subjects. As Blackmore noted (p. 177), Siegel referred to aerial memories when he wrote:

Common complex images included childhood memories and scenes associated with strong emotional experiences that the subjects had undergone. These hallucinatory images were more than pictorial replicas; many of them were elaborated and embellished into fantastic scenes. This constructive aspect of imagery can be illustrated by a simple exercise. Recall the last time you were swimming in the ocean. Now ask yourself if this memory includes a picture of yourself running along the beach or moving about in the water. Such a picture is obviously fictitious, since you could not have been looking at yourself, but images in the memory often include fleeting pictures of this kind. Our subjects often reported equally improbable images, such as aerial perspectives and underwater views. . . . The subjects frequently reported feeling dissociated from their bodies. (p. 136)

So there we have it: Blackmore's dying brain hypothesis needs the bird's-eye view to explain OBEs; and the bird's-eye view proposition is predicated on Nigro and Neisser's article, which they wrote described a preliminary study based on an unrepresentative sample, and in which observer experiences may account for many observer memories.

Blackmore's OBE explanation also rested on Siegel's 1977 article devoted to hallucinations, in which one paragraph discussed tangentially the bird's-eye perspective. That paragraph gave Siegel's opinion on the aerial perspective, but cited no data and no studies on memory reconstruction, such as Nigro and Neisser conducted. In fact, the research reported in Siegel's article was on hallucinations, which raises the next objection to the Siegel citation. Blackmore committed a fallacy of logic, the appeal to authority, by citing an authority in one area-hallucinations-as an authority in another-memory reconstruction.

In addition, Siegel seems to have confused observer memories, such as seeing oneself running along a beach, with observer experiences, such as "childhood memories and scenes associated with strong emotional experiences," concluding, in effect, that these are all observer 
memories. Furthermore, it is possible that some of Siegel's subjects were abused as children, and these repressed memories and feelings were then released by their taking hallucinogenic drugs administered to them by Siegel. Also, the dissociation he mentioned (p. 136) could have been, in some of the cases, OBEs due to the administered drugs rather than what he called "improbable images, such as aerial perspectives."

The case for the bird's-eye view in an OBE based on the Nigro and Neisser article and Siegel's opinion is very tenuous indeed. It would seem that if Blackmore wanted to argue that the OBE is caused by a breakdown of body image with a subsequent mental reconstruction of what happened to the person while unconscious, she would have a more cogent reductionist argument by saying the OBE is due to the tactile and auditory input still perceivable to the unresponsive NDEr.

In this regard, she cited Michael Sabom's patient who, while out of his body, saw "a shot" being administered to him near his groin. Sabom wrote that in fact blood was being withdrawn from this NDEr's femoral artery. Sabom felt the mistake in his patient's interpretation was understandable because the NDEr did not hear anything during his experience. Blackmore suggested that the man never left his body, but felt the pain of the needle stick while semiconscious and later drew the conclusion that he had been given an injection (pp. 124-125).

If reconstruction of stimuli from other senses is indeed the case, then why do we not find out-of-body perceptions from a supine or prone or even a sitting position, at least occasionally? Using Blackmore's explanations and given the preliminary nature of Nigro and Neisser's study, which in any event found that field memories-that is, memories from the original perspective-occur more frequently than observer memories, one would expect NDErs to say that during an OBE they looked up to see living relatives and/or medical providers.

Blackmore did present a case (pp. 227-228) of a man who said that as he was being wheeled into an operating room he thought he saw welcoming angels surrounded by light, but later "he was quite sure that they were the doctors and nurses waiting for him in the theatre" (p. 227). Blackmore did not mention whether or not the man's perspective was a bird's-eye view. Even though the man had had more than one cardiac arrest prior to this surgery, it is not clear from Blackmore's account that this man was having an NDE at the time 
of his seeing the "angels/medical providers" or that he was even unresponsive. It is, however, the kind of reconstruction one might expect from a semiconscious person given preoperative anesthesia: a mental reconstruction of the sensory input prior to surgery.

In any event, the out-of-body perceptions of NDErs are far more likely to be similar to the following two cases, which I share with the reader knowing full well Blackmore's argument that they again do not offer incontrovertible proof of OBEs. Having floated out of her body and looking down on the operating room, one of my interviewees noticed the surgeon working on her was not the doctor she had authorized for her operation. She told me her attorney later discovered her medical records were missing, with the two doctors involved acknowledging off the record in a private meeting that her out-ofbody perception was accurate.

Another respondent had an OBE during a sexual assault while running on a beach. The perpetrator punched her repeatedly in the face and then strangled her during the assault, at which time she floated out of her body to about 15 feet above the attack site. She was able to describe accurately the escape path taken by her assailant. She saw him from behind running down a path she had never seen before. She told me she had described in court seeing the assailant leave and the direction and path he took, but was never asked by the defense attorney how she could accurately describe these details when she was supine, on the ground, and unresponsive. The path she described was confirmed by the police reports, and the man was convicted of the assault.

In summary, then, Blackmore's OBE explanation "as part of the mind's own creations as it tries to make sense of the physical struggle going on between life and death" (p. 227) just does not seem to hold up, given the tenuous nature of the work of Nigro and Neisser and of Siegel, on which it is based, and the prediction that a mental reconstruction of memories in which field memories predominate should yield at least some, if not many, sightings from a supine or prone perspective.

\section{Unconditional Love}

The second question I pose for Blackmore is how the dying brain hypothesis explains the overwhelming feelings of unconditional love experienced during some NDEs. 
Blackmore wrote that "The joy and peace are consistent because of the natural opiates released under stress" (p. 261). She explicated the role of endorphins and enkephalins for pain relief, and wrote that "Endorphins cause just the kinds of emotional response, including pleasure, joy, calmness and freedom from pain, that occur in NDEs" (p. 108). But she never gave a clear, reductionist explanation for the sense of unconditional love reported by NDErs.

As Kenneth Ring wrote, the NDEr may enter "a magnetic and brilliant golden light, from which emanates [sic] feelings of love, warmth, and total acceptancen $(1980$, p. 103). Cherie Sutherland wrote that NDErs may encounter "a Being of Light who radiates love" (1993, p. 244). Raymond Moody and Paul Perry wrote that "the Being radiates total love" (1988, p. 10). And Moody noted (1975, p. 59):

The love and the warmth which emanate from this being to the dying person are utterly beyond words, and he feels completely surrounded by it and taken up in it, completely at ease and accepted in the presence of this being.

One of Sutherland's interviewees said:

I was going towards a very bright light. And as I was travelling along ... I got stopped, just stopped before I got to the light. And I felt this extreme presence of love, just absolute love. (1992, p. 10)

Melvin Morse wrote:

Most of the patients I have spoken to describe it [the Light] essentially as a pure light of unconditional love. Others call it "all-knowing," "all-forgiving," and "all-loving." (Morse and Perry, 1990, p. 116)

One woman who recalled a childhood NDE remarked that the light represented love: it "had the feeling of unconditional love" (Morse and Perry, 1990, p. 116).

Margot Grey also noted "a love and a beauty that surpasses anything known on this earth" (1985, p. 49). One NDEr she interviewed said:

Love is the major impression I still retain. In heaven there is light, peace, music, beauty and joyful activity, but above all there is love and within this love I felt more truly alive than I have ever done before. (1985, p. 53)

Another of Grey's respondents said about his NDE:

it's absolute pure love. ... What the light communicates to you is a feeling of true, pure love. You experience this for the first time ever. You can't compare it to the love of your wife, or the love of your children or sexual love. Even if all those things were combined, 
you cannot compare it to the feeling you get from this light. (1985, pp. 53-54)

Two NDErs I interviewed made similar comments. One, who had an NDE from a heart attack, said that during his experience he encountered "a diaphanous Christ-like figure" with outstretched hands emanating a loving light. He then knew how well-loved he was due to this figure. He could feel the love, and he wanted to be enveloped by that love.

The other NDEr, "Joan," about whom I have written elsewhere (Serdahely, 1993) said the bright white light for her was love that she brought back with her. It was a love that "circulated" through her entire being.

Given the consistent reports of an experience of love during an NDE, the dying brain hypothesis needs to address from where in the brain these feelings of unconditional love arise: which neurotransmitters and/or cortical structures are responsible for this love? Blackmore herself noted that "Many positive NDEs begin with nothing but [a] sense of peace and love and some never get further than this" (p. 104), but she did not offer a materialistic explanation for the unconditional love during an NDE.

A similar question can be asked with respect to distressing NDEs: what are the anatomy and physiology of distressing NDEs? Dying to Live never gave a clear explanation in its section on hellish experiences; Blackmore only cited one case of a man who had been given an injection of naloxone, and claimed that the naloxone changed his near-death experience from pleasant to horrific. But clearly no naloxone injections were administered in most of the published cases of hellish or distressing NDEs. So we are left wanting a reductionist explanation here, too.

\section{Individual Variation in NDEs}

The third question I pose for Blackmore is how the reductionist brain model explains the observation that NDEs vary from person to person, often greatly, in their phenomenology.

Blackmore differentiated between consistency and invariance for NDEs:

We have consistency but not invariance. Yes, the NDE is universal in the sense that something like the modern NDE has been reported 
in adults and children in many ages and cultures. And, 'no,' it is not always the same but varies with the individual, the culture and the circumstances. (p. 22)

We have explored many different kinds of NDEs and seen that, although no two are the same, there are consistent patterns: the tunnel; the light; the out-of-body experience; the return to life and the changes it brings. (p. 261)

While Blackmore used "consistency" to support the dying brain hypothesis, she never addressed how the brain then generates the myriad variations within those patterns. How does the dying brain hypothesis account for the fact that NDEs vary "with the individual, the culture and the circumstances" (p. 22)?

With respect to the consistent patterns, Blackmore wrote that the joy and peace are due to endorphins; the tunnel, light, and noises a result of anoxia; the $\mathrm{OBE}$ due to a change in body image and sensory deprivation; and the life review from endorphin-induced temporal lobe and limbic system seizures. But within each of these patterns are countless variations, whether in affective tone, during the tunnel experience, within the light, or during OBEs. As she wrote, "no two [NDEs] are the same" (p. 261). But these many specific differences within each pattern are left unexplained by the dying brain hypothesis.

For example, if the life review is due to endorphins, then why are there so many varieties of life review? Some people see the life review chronologically, some see all events simultaneously, and some see only significant events. Some see the life review by themselves, and others with a loving being or occasionally with other beings. Most feel no judgment, while some do. Some experience empathy, while others do not. Some see it as watching a movie, others as countless television screens, and yet others as snapshots; one of my respondents saw several blank or "undeveloped photos" of her life, which was portrayed during her life review as a series of photographs. Two of my respondents had lives reviews, seeing past as well as present lives; while most NDErs only review their present life. How does the dying brain hypothesis explain such great variation within the basic pattern of the life review?

A similar argument can be made for the variations within each of the basic patterns Blackmore identified: the tunnel; the light; the affective tone: and the OBE. If the NDE is only a creation of the brain, then why wouldn't the brain be programmed to release an 
experience that is not only consistent but also invariant within each pattern, from person to person, culture to culture, and time to time? Wouldn't invariance more likely be the rule, if the NDE is actually an outcome of evolution to insure the survival of the species, as Blackmore implied in the Preface of her book?

Siegel (1977), cited by Blackmore as noted above, argued that hallucinations are a universal phenomenon, and, of course, generated by the brain. He cited Heinrich Klüver's delineation of four "simple form constants" that appear in the first stage of hallucinations ( $p$. 132). Siegel called the second stage "complex images," and wrote:

One would expect the forms and scenes of complex imagery to be almost infinitely diverse. Actually constants appear even at this stage. (p. 132)

So here we have brain-generated phenomena that Siegel found were not infinitely diverse but quite constant, that is, invariant for both stages. If NDEs are indeed products of the brain, then why wouldn't they also be invariant like hallucinations?

Consistency, as Blackmore pointed out, does not prove an afterlife. Consistency is what one would expect from either the dying brain or the afterlife hypothesis. Variance within the patterns identified by Blackmore, while seemingly incompatible with the dying brain hypothesis, is just what one would expect with the afterlife hypothesis. I have proposed what I call the "individually tailored hypothesis" (Serdahely, 1995), which does account for the variation and individualization of each NDE within the consistent patterns of $\mathrm{OBE}$, tunnel, and light. The individually tailored hypothesis suggests that the innumerable variations within the NDE are due to experiencers getting what they need from the experience in a way they can accept it, in order to facilitate their own psychospiritual growth.

\section{Separation of the "True Self" From the Physical Body}

My final question for Blackmore is whether neural disinhibition could possibly be a causative factor in the separation of the "true self," spirit, or soul, from the physical body.

A case was put forward in Dying to Live that anoxia can lead to the disinhibition and subsequent excitation of neurons, especially in the visual cortex and perhaps in the temporal lobes. Blackmore also argued that endorphins cause neural disinhibition and consequent 
seizures in the temporal lobes and the limbic system. The neural disinhibition due to anoxia and endorphins, then, according to the dying brain hypothesis, is responsible for what we call the NDE. Blackmore pointed out that certain drugs, in particular depressants, may damp down an NDE by increasing neural inhibition.

I recently interviewed a man who tried to commit suicide by drinking a massive dose of animal tranquilizer, available to him in his work, along with alcohol. His NDE was dampened with the imagery being less clear or fuzzier for him than the usual vividness of an NDE. This case was very similar to one reported by Ring (1980, pp. 122-24) in terms of its overall pattern and tone, but differs with respect to the specifics of the NDE.

Given her materialist position, Blackmore, of course, was unwilling to entertain the possibility that there is such a thing as what she called a soul (I prefer the term "true self"), let alone that the soul might leave the body, a paradigm shift I am willing to make, given the discussion of OBEs above. She asked, "Why . . . should disinhibition and random excitation produce NDEs?" (p. 66), and she answered that question with a materialist response.

However, it is possible that the disinhibition she described in the visual cortex and especially in the temporal lobes with its concomitant neural excitation may be the physiological condition that "opens the gate," so to speak, to release the soul from the physical body. After all, if there is a soul, then it has to interface with the physical body somehow.

Blackmore wrote that there are other conditions known to cause cortical disinhibition or excitation, including some drugs, especially hallucinogens like LSD, hashish, and mescaline; epilepsy and temporal lobe seizures; some brain chemicals; and, she implied, conditions like meditation, fasting, prayer, fatigue, and dreams. Many of these conditions have also been associated with NDEs and/or OBEs. Therefore, it is possible, given our current understanding of the temporal lobe, that neural disinhibition could possibly lead to the separation of the "true self" from the physical body.

I have one more question to pose while we are considering neural disinhibition. As mentioned above, Blackmore attributed the tunnel, light, and noises to disinhibition. With that as the premise, my question is how the dying brain hypothesis accounts for seeing deceased relatives during an NDE. If relatives are encountered in the course of an NDE, almost always they are said to be deceased. So how does the disinhibited brain know to call up these memories only, and not 
memories or images of living loved ones? It would seem that if the brain alone is responsible for an NDE, then it is more likely that the brain would recall images of living loved ones to provide the comfort and assurance that NDErs report from encountered deceased beings.

For example, when Patrick had an NDE at age 7, he encountered his two deceased pets, even though all the people close to him were still alive (Serdahely, 1989-90). How did his disinhibited brain know how to do that? Or if there is a random firing of disinhibited neurons, then why do these neurons almost always produce images of deceased loved ones?

Perhaps to answer this question Blackmore would cite the case, discussed above, of the man being wheeled into the operating room, arguing that the semiconscious or unresponsive person's brain interprets encounters with living relatives and medical providers as deceased beings. Again, it is not clear from Blackmore's account of this case (p. 227) that this man was having an NDE or was even unresponsive. But even if that were so, then the dying brain hypothesis needs to explain how and why the brain interprets these entities almost always as being deceased.

\section{Conclusion}

Blackmore concluded that "If evidence changes in the future and truly convincing paranormal events are documented then certainly the theory I have proposed will have to be overthrown" (p. 262). The four questions I have posed here do not, of course, provide such evidence, but they do present some formidable challenges to the dying brain hypothesis.

\section{References}

Blackmore, S. (1993). Dying to live: Near-death experiences. Buffalo, NY: Prometheus. Grey, M. (1985). Return from death: An exploration of the near-death experience. London, England: Arkana.

Moody, R. A. (1975). Life after life. Covington, GA: Mockingbird Books.

Moody, R. A., and Perry, P. (1988). The light beyond. New York, NY: Bantam.

Morse, M., and Perry, P. (1990). Closer to the light: Learning from the near-death experiences of children. New York, NY: Villard.

Nigro, G., and Neisser, U. (1988). Point of view in personal memories. Cognitive Psychology, 15, 467-482. 
Ring, $\mathrm{K}$ (1980). Life at death: A scientific investigation of the near-death experience. New York, NY: Coward, McCann and Geoghegan.

Serdahely, W. J. (1989-90). A pediatric near-death experience: Tunnel variants. Omega, $20,55-62$.

Serdahely, W. J. (1992). Similarities between near-death experiences and multiple personality disorder. Journal of Near-Death Studies, 11, 19-38.

Serdahely, W. J. (1993). Near-death experiences and dissociation: Two cases. Journal of Near-Death Studies, 12, 85-94.

Serdahely, W. J. (1995). Variations from the prototypic near-death experience: The "individually tailored" hypothesis. Journal of Near-Death Studies, 13, 185-196.

Siegel, R. (1977). Hallucinations. Scientific American, 237(4), 132-140.

Sutherland, C. (1992). Transformed by the light: Life after near-death experiences. Sydney, Australia: Bantam.

Sutherland, C. (1993). Within the light. Sydney, Australia: Bantam. 
